\title{
Minimal invasive procedures for the treatment of genitourinary syndrome of menopause (GSM). Un update
}

\section{Dimitrios Zacharakis, Eleni Pitsouni, Themos Grigoriadis, Christos Kalantzis, Dimitrios Loutradis, Stavros Athanasiou}

$1^{\text {st }}$ Department of Obstetrics and Gynecology, National and Kapodistrian University of Athens, "Alexandra" Hospital, Athens, Greece

\section{Corresponding Author}

Dimitrios Zacharakis, MD, $1^{\text {st }}$ Department of Obstetrics and Gynecology, "Alexandra" General Hospital, University of Athens, 80 Vas. Sofias Av., 11528, Athens, Greece, Tel.: +30 6976403626, Fax: +30 2107295339, e-mail: dimzac@hotmail.com

\section{Abstract}

Genitourinary syndrome of menopause (GSM) is a new term for a condition more renowned as atrophic vaginitis. It is used to describe a variety of menopausal symptoms and signs that are related to the physical changes of the vulva, vagina and lower urinary tract. The etiology of GSM is secondary to decreased levels of endogenous estrogens and represents a common but underreported condition. In this update we present novel minimal invasive procedures that may benefit GSM symptoms making particular focus on platelet-rich plasma (PRP) treatment, hyaluronic acid (HA) treatment and energy-based devices $\left(\mathrm{CO}_{2}\right.$ laser, YAG laser, radiofrequency device and high intensity focused ultrasounds).

Currently, PRP has becoming popular as a non-operative treatment option for GSM symptoms. The principle underlying PRP treatment has to do with its ability to reproduce natural mechanisms of cell regeneration through the sequential secretion of numerous growth factors. Moreover, HA has been widely used as an important ingredient in topical hydrating and lubricating gels or injected for conditions such as dyspareunia. Lately, it has also been used as a new tool to treat age-related fat lysis of the female genitalia and especially the labia majora. The Energy based devices have been recently suggested for the management of GSM. They utilize technologies such as laser, electromagnetic waves and high intensity focused ultrasounds to focus energy in the vaginal wall heating the targeted tissue at various depths without causing any pain or needing a recovery time. These minimal invasive procedures appear to be promising options for the treatment of GSM symptoms. However, further studies are necessary to evaluate more thoroughly the effectiveness and safety of these procedure and assess medium and long term results.

Key words: GSM, Laser, Hyaluronic acid, PRP, Dyspareunia, Vaginal atrophy. 


\section{Introduction}

Genitourinary syndrome of menopause (GSM) is a new term for a condition more renowned as atrophic vaginitis. It is used to describe a variety of menopausal symptoms and signs that are related to the physical changes of the vulva, vagina and lower urinary tract ${ }^{1}$. The GSM includes genital (dryness, burning, irritation), sexual (lack of lubrication, discomfort, dyspareunia) and urinary symptoms (urgency, dysuria, recurrent urinary tract infections) ${ }^{1}$.

The etiology of GSM is secondary to decreased levels of endogenous estrogens ${ }^{2}$ and represents a common and underreported condition due to sexual embarrassment or general disregard due to associating it as a liability of natural aging ${ }^{2}$. The syndrome or its features manifest in approximately $15 \%$ of premenopausal $^{3}$ and $40-54 \%$ of postmenopausal women ${ }^{4}$.

Clinicians play a major role in recognizing the signs of GSM because many women are reluctant to report their symptoms. GSM is most commonly diagnosed when the patient presents with dyspareunia ${ }^{5}$. In fact when the vulvo-vaginal epithelium is inadequately lubricated, ulceration and fissures may develop during intercourse, causing dyspareunia secondary to vaginal dryness. Vaginismus, or painful spasm of vaginal muscles, can also occur as a physiological response when there is anxiety toward expected sexual pain ${ }^{5}$.

The symptoms of GSM are particularly bothersome and usually require intervention, as vaginal health represents an essential component of active and healthy aging in women at midlife and beyond. In the literature, various hormonal products or vaginal lubricants or moisturizers are used in order to relieve patient's symptoms ${ }^{5}$. In this update we present novel minimal invasive procedures that may benefit GSM symptoms making particular focus on platelet-rich plasma (PRP) treatment, hyaluronic acid (HA) treatment and energy-based devices $\left(\mathrm{CO}_{2}\right.$ laser,
YAG laser, radiofrequency device and high intensity focused ultrasounds).

\section{Platelet-rich plasma (PRP) treatment}

PRP is an autologous solution of human plasma that has an increased content of platelets (4-7 times the baseline concentration) ${ }^{6}$. The principle underlying PRP treatment has to do with its ability to reproduce natural mechanisms of cell regeneration through the sequential secretion of numerous growth factors (platelet-derived growth factors, transforming growth factors $b$, epithelial growth factors, vascular endothelial growthfactor, insulin-like growth factor, basic fibroblast growth factors), which modulate cell proliferation, differentiation, angiogenesis and chemotaxis ${ }^{7-9}$.

The PRP preparations can be classified according to the preparation method and the content of the PRP sample. Preparations vary in terms of centrifugation speed, centrifugation time, and anticoagulant use, while the content varies depending on the predominant constituent (e.g., platelets, leukocytes, or growth factors) ${ }^{10-11}$.

Currently, PRP has becoming popular as a nonoperative treatment option for a broad spectrum of gynecological disorders. Based on the experience of using PRP to improve smoothness and to decrease wrinkles in the facial area ${ }^{12-13}$, aesthetic practitioners have also used PRP for the regeneration of the vaginal mucosa and skin in patients with vaginal atrophy and symptoms of dyspareunia. Following PRP injection, vaginal vascularity seems to increase with a subsequent improvement of sexual function. In addition, the mucosa becomes thicker and firmer, making the vagina look much more youthful ${ }^{14-15}$.

A few pilot studies concerning GSM treatment using PRP have reported promising findings ${ }^{15-17}$. Kim et $\mathrm{al}^{15}$ reported the use of autologous lipofilling mixed with PRP in a 67 year-old patient with vaginal atrophy. A total of $40 \mathrm{cc}$ of autologous fat mixed with 
PRP was transferred to labia majora. Lipofilling with PRP relieved symptoms, restored contour of the labia majora and achieved remission of lichen sclerosus on labia minora.bRegarding use of PRP in sexual function improvement Runels et $\mathrm{al}^{17}$ injected PRF in clitoris and vagina of 11 women aged between 24-64 with female orgasmic disorder, hypoactive sexual arousal disorder, anorgasmia or dyspareunia. 71\% of the study population improved their status from being "distressed" to being "not distressed" after the procedure, while two patients (18\%) showed no change in their levels of distress.

In conclusion, PRP is an innovative, affordable and easily performed therapeutic modality, which has been recently studied in women with various urogenital disorders. Although the specific mechanisms of action of PRP have not been completely elucidated, various studies have shown that the high concentration of growth factors in PRP may potentially initiate inflammatory reactions and promote the healing process ${ }^{10-11}$. Efficacy appears promising and the risks of PRP therapy seem to be minimal. However, evidence is based on a few low-quality pilot studies ${ }^{15-17}$, case reports and anecdotal series, including only a small number of patients and with a short-term follow-up. Randomized controlled studies based on validated objective and patients reported outcomes are urgently required to confirm its efficacy and safety.

\section{Hyaluronic Acid (HA) treatment}

HA is a high-molecular-weight anionic polymer. It is a member of the non-sulfated glycosaminoglycan superfamily, and its structure is based on the linear repetition of disaccharide sequences ${ }^{18}$. It is naturally present in many tissues and plays important physiologic roles in living organisms, including maintenance of the viscoelasticity of liquid connective tissues and control of tissue hydration, especially in the dermis, mucous membranes, and submucosal tissue layers ${ }^{18}$.
In gynecology, HA has been widely used as an important ingredient in topical hydrating and lubricating gels or injected for conditions such as dyspareunia. Lately, it has also been used as a new tool to treat age-related fat lysis of the female genitalia and especially the labia majora ${ }^{19-20}$. The labia majora are anatomic structures primarily dedicated to protection and shock absorption and therefore labia majora fat is different from facial fat. Thus injected HA gel characteristics should be adapted to this specific environment to prevent product migration after the treatment ${ }^{18}$. In Europe, only one product (Desirial Plus, Vivacy) has been clinically tested and received approval for this very specific indication.

In the literature there are several studies suggesting that HA may be useful in the treatment of GSM symptoms. However, these studies are small and lack rigor. Aguilar et $\mathrm{al}^{19}$ presented a case of a 39 years old woman who had vaginal laxity resistant to physical therapy, a retractile episiotomy scar and a slightly atrophic vaginal mucosa. She was treated with injection of purified fat cells in the posterior vaginal wall and PRP-HA solution in the perineum focusing in the episiotomy. During the follow-up period the authors observed an improvement of the patients sexual life and vulvoperineal rejuvenation. Moreover, Hersant et al ${ }^{16}$ studied the efficacy of PRP combined with hyaluronic acid for the treatment of vulvovaginal atrophy in postmenopausal women with history of breast cancer. All participants (20 women) showed improvement in the clinical symptoms of vaginal dryness and dyspareunia. Improvement in hydration and vaginal epithelial integrity was reported. No adverse events were reported. The authors concluded that the injection of PRP combined with hyaluronic acid is a promising method to improve the trophicity and hydration of vaginal mucosa in postmenopausal breast cancer survivors with contraindications to hormone therapies. 


\section{Energy Based Devices}

Energy based devices have been recently suggested for the management of GSM ${ }^{21-26}$. They utilize technologies such as laser, electromagnetic waves [Radiofrequency (RF)] and high intensity focused ultrasounds (HIFU) to focus energy in the vaginal wall heating the targeted tissue at various depths without causing any pain or needing a recovery time ${ }^{21}$. The principle of these treatments is the induction of neocollagenesis, vascularization, lubrication and tightness of vaginal structures ${ }^{21}$. However, there is an FDA warning regarding all these devices because they have not yet obtained an FDA approval for vaginal rejuvenation and there is ongoing research on this field ${ }^{27}$.

\section{Lasers}

Lasers were the first to come out with many published studies in PubMed evaluating their safety and efficacy using objective and subjective outcomes ${ }^{25-47}$. There are 2 types of laser-devices; the $\mathrm{CO}_{2}$ and Er:YAG devices ${ }^{21}$. These 2 laser types differ in wavelength, fluence, duration of the laser pulse, spot size and penetration depth ${ }^{21}$. The standard treatment protocols are 3 laser-sessions at monthly intervals for both laser types. For $\mathrm{CO}_{2}$-laser this standard protocol may be extended by an additional $4^{\text {th }}$ or even a $5^{\text {th }}$ treatment ${ }^{35}$. Nevertheless, all studies, regardless of laser type, consistently suggest that a significant improvement in all GSM symptoms and clinical signs is present following laser treatments ${ }^{25-47}$. Specifically, dyspareunia, vaginal dryness, itching, burning, dysuria, frequency, urgency and incontinence could be significantly decreased as soon as 1-month after the first laser application ${ }^{45-47}$. This positive impact of laser treatments was further reflected by improvements in women's quality of life and sexual function ${ }^{30,35,38-42,45-47}$. It is essential that all aspects of sexual function (desire, arousal, satisfaction, orgasm and lubrication), as assessed by validated patient reported outcomes, were significantly improved ${ }^{30,35,38-42,45-47}$. Specifically, it has been found that following 3,4 or $5 \mathrm{CO}_{2}$ lasertreatments normal sexual function resumed in 41\%, $69 \%$ and $84 \%$ of women respectively ${ }^{35}$. Moreover, lasers' positive results were maintained for a longterm (up to 36-months) ${ }^{30,32,39-41}$. In fact, 1-year after the last laser-session, improvements in dyspareunia, vaginal dryness and all aspects of sexual function were the same as 1-month after the last laser ses$\operatorname{sion}^{30}$. The authors suggested that 1-month after the last laser-therapy may be indicative of the GSM symptoms at 12 -months and the time point to decide whether treatments' extension should be offered $\left[{ }^{30}\right]$. Randomized controlled trials suggested that $\mathrm{CO}_{2}$ laser had a similar efficacy as vaginal estrogens in improving all GSM symptoms and a better efficacy than promestriene or lubricants in improving vaginal health ${ }^{28,29,33,34}$.

Cytological, microbiological and histopathological studies further confirmed the efficacy of lasertreatments indicating that the "placebo effect" is probably not the mode of lasers' action $37,38,42,48-51$. The pathophysiological changes of the vaginal mucosa resembled to those at premenopausal status. The international Society for the Study of Women's Sexual Health and the North American Menopause Society have suggested that the increased parabasal cells on maturation index and $\mathrm{pH}$ values of the vaginal fluid $>4.5$ are supportive findings of the GSM diagnosis ${ }^{1}$. Studies evaluating these 2 aspects following $\mathrm{CO}_{2}$-laser treatments, found that the vaginal maturation value increased significantly with a profound decrease of parabasal cells, while $\mathrm{pH}$ of the vaginal fluid reached values $<4.5^{37,38,42}$. In accordance, $\mathrm{CO}_{2}$-laser treatments proved to be beneficial for the vaginal microenvironment, as lactobacilli increased significantly and uropathogens decreased significantly, resulting in a significant increase of women with normal vaginal flora ${ }^{48}$. Moreover, authors of histopathological studies stated that there was a restoration of the vaginal 
thick squamous stratified epithelium with a significant storage of glycogen in the epithelial cells and a high degree of glycogen-rich shedding cells at the epithelial surface ${ }^{21,49-51}$. In addition, they observed active fibroblasts, collagen and ground substance molecules as well as newly-formed papillae of connective tissue and blood capillaries penetrating the papillae providing all the necessary metabolic support $^{21,49-51}$.

\section{Radiofrequency (RF) devices}

$\mathrm{RF}$ devices emit within a frequency range of $3 \mathrm{kHz}$ to $24 \mathrm{GHz}$ generating an electric field that induces collisions and motion among charged and polar atoms and molecules ${ }^{21,26}$. Energy is transferred to the tissue as heat ${ }^{21,26}$. RF devices for vulvovaginal application include temperature-controlled devices, cryogen-cooled monopolar devices, multipolar radio frequency-pulse electromagnetic fields devices and focused radio-frequency devices. The mechanism of action and treatments protocols between these RF types differ substantially. For example, the temperature-controlled devices produce a transmucosal heating of the tissue to $40-47^{0}$ promoting tightening of the vaginal mucosa at $460 \mathrm{kHz}^{21,26}$. Three treatments are suggested at 4-6 weeks intervals up to 30 minutes time per area with a maintenance every 6 months ${ }^{21,26}$. The cryogen-cooled monopolar $\mathrm{RF}$ reverse thermal gradient and cools the surface mucosa allowing a high RF energy at $6 \mathrm{MHz}$ promoting neocollagenesis ${ }^{21,26}$. In this RF type of device, one treatment with an annually maintenance is adequate for clinical results to be observed ${ }^{26}$. The multipolar RF and pulsed electromagnetic fields produce a non-thermal release of tissue growth factors (FGF2) to trigger new collagen synthesis ${ }^{26,52}$. Only one treatment is suggested but with unknown maintenance time ${ }^{26}$. The focused RF device requires 2-4 treatments at 2-3 weeks intervals annually with results to be obtained after 4 weeks of treatment ${ }^{26}$.
Regardless the type of RF device, studies have consistently reported that dyspareunia, vaginal dryness, sexual function (especially arousal and orgasm) and satisfaction, frequency, urgency, incontinence and vulvovaginal tightening may improve significantly following RF treatments ${ }^{21,26,53-57}$. However, only 6 studies including 120 participants have been published on the field; one of them was randomized placebo-controlled trial. All but 2 studies had a short-term duration; 1 study presented results at 6-months and 1 study at 12-months duration suggesting a sustained positive efficacy.

\section{High intensity focused ultrasound (HIFU)}

HIFU bases its action on a focused thermal effect at a certain depth producing cavitation, apoptosis and thermal coagulative microdamage caused by a $60-70^{\circ} \mathrm{C}$ temperature. This is a technique originally used in oncology for tumor removal ${ }^{58}$. This action is determined by the concentration of energy emitted by a round and concave ultrasound transducer of $20 \mathrm{~mm}$ in diameter that directs convergent ultrasonic waves to a specific focal depth according to the position of the transducer crystal. These convergent mechanical waves produce the molecular vibration of the water, which raises the temperature of the tissue and produces its physical modification $s^{58}$.

Manufactures advocate that HIFU produces a better rejuvenation effect than laser and $\mathrm{RF}$ due to its capability to perform a deeper penetration depth into fibromascular layer up to adventitia, while lasers may reach up to lamina propria and $\mathrm{RF}$ up to fibromascular layers ${ }^{58}$. Currently there are no published studies evaluating its safety and efficacy in postmenopausal women with GSM.

\section{References}

1. Portman DJ, Gass ML. Vulvovaginal Atrophy Terminology Consensus Conference Panel. Genitourinary syndrome of menopause: new terminology 
for vulvovaginal atrophy from the International Society for the Study of Women's Sexual Health and the North American Menopause Society. Menopause 2014; 21: 1063-1068.

2. Mac Bride MB, Rhodes DJ, Shuster LT. Vulvovaginal atrophy. Mayo Clin Proc 2010;85:87-94.

3. Palacios S. Managing urogenital atrophy. Maturitas 2009;63:315-8.

4. DiBonaventura M, Luo X, Moffatt M, Bushmakin AG, Kumar M, Bobula J. The association between vulvovaginal atrophy symptoms and quality of life among postmenopausal women in the United States and Western Europe. J Womens Health (Larchmt) 2015;24:713-22.

5. Gandhi J, Chen A, Dagur G, Suh Y, Smith N, Cali B, Khan SA. Genitourinary syndrome of menopause: an overview of clinical manifestations, pathophysiology, etiology, evaluation, and management. Am J Obstet Gynecol. 2016;215:704-711.

6. Marx RE. Platelet-rich plasma (PRP): what is PRP and what is not PRP? Implant Dent 2001;10:225-8.

7. Conley CL. Hemostasis. Mountcastle VB, editor. Medical Physiology. St. Louis: The C.V. Mosby Company; 2004. p. 1137-46.

8. Lubkowska A, Dolegowska B, Banfi G. Growth factor content in PRP and their applicability in medicine. J Biol Regul Homeost Agents 2012; 26 (2 Suppl 1):3s-22s.

9. Marx RE. Platelet-rich plasma: evidence to support its use. J Oral Maxillofac Surg 2004; 62: 489-96.

10. Dohan Ehrenfest DM, Andia I, Zumstein MA, Zhang CQ, Pinto NR, Bielecki T. Classification of platelet concentrates (platelet-rich plasma-PRP, plateletrich fibrin-PRF) for topical and infiltrative use in orthopedic and sports medicine: current consensus, clinical implications and perspectives. Muscles Ligaments Tendons J 2014;4:3-9.

11. Dohan Ehrenfest DM, Bielecki T, Mishra A, Borzi- ni P, Inchingolo F, Sammartino G, et al. In search of a consensus terminology in the field of platelet concentrates for surgical use: platelet-rich plasma (PRP), platelet-rich fibrin (PRF), fibrin gel polymerization and leukocytes. Curr Pharm Biotechnol 2012;13:1131-7.

12. Zhang M, Zhou B, Luo D.: Applications and efficacy of platelet rich plasma in dermatology. A clinical review. J Cosmet Dermatol. 2018;00:1-6

13. Michael S Leo.Bs: Systematic review of the use of platelet rich plasma in aesthetic dermatology. Journal of cosmetic Dermatology,2015; 14,315-323

14. Ruth Styles. Would you plump up your vagina with fillers? Top cosmetic doctor warns of risky new trend after being inundated with clients looking for 'genital enhancements' [Internet]. London: Daily Mail; 2015 [cited 2018 Jun 17].

15. Kim SH, Park ES, Kim TH. Rejuvenation using platelet-rich plasma and lipofilling for vaginal atrophy and lichen sclerosus. J Menopausal Med 2017;23:63-8.

16. Hersant B, SidAhmed-Mezi M, Belkacemi Y, Darmon F, Bastuji-Garin S, Werkoff G, Bosc R, Niddam J, Hermeziu O, La Padula S, Meningaud JP. Efficacy of injecting platelet concentrate combined with hyaluronic acid for the treatment of vulvovaginal atrophy in postmenopausal women with history of breast cancer: a phase 2 pilot study. Menopause. 2018;25(10):1124-1130.

17. Runels C, Melnick H, Debourbon E, Roy L. A pilot study of the effect of localized injections of autologous platelet rich plasma (PRP) for the treatment of female sexual dysfunction. J Womens Health Care 2014;3:169

18. Female cosmetic genital surgery: Concepts, Classification and Techniques. Hamori CA., Banwell P., Alinsod R. New York : Thieme, [2017]

19. Aguilar P, Hersant B, SidAhmed-Mezi M, Bosc R, Vidal L, Meningaud JP. Novel technique of vulvo- 
vaginal rejuvenation by lipofilling and injection of combined platelet-rich-plasma and hyaluronic acid: a case-report. Springerplus. 2016 26;5:1184.

20. Origoni M, Cimmino C, Carminati G, Iachini E, Stefani C, Girardelli S, et al. Postmenopausal vulvovaginal atrophy (VVA) is positively improved by topical hyaluronic acid application: a prospective, observational study. Eur Rev Med Pharmacol Sci. 2016;20:4190-4195.

21. Tadir Y, Gaspar A, Lev-Sagie A, Alexiades M, Alinsod R, Bader A, et al. Light and energy based therapeutics for genitourinary syndrome of menopause: Consensus and controversies. Lasers Surg Med 2017;49:137-59.

22. Franic D, Fistonic I. Laser Therapy in the Treatment of Female Urinary Incontinence and Genitourinary Syndrome of Menopause: An Update. Biomed Res Int. 2019;2019:1576359

23. Miller MD. The vaginal wellness consultation for the treatment with energy-based devices. Semin Cutan Med Surg 2018;37:233-5

24. Alexiades M. Device-based treatment for vaginal wellness. Semin Cutan Med Surg 2018;37:226-232

25. Faubion SS, Larkin LC, Stuenekel CA, Bachmann GA, Chism LA, Kagan R, et al. Management of genitourinary syndrome of menopause in women with or at high risk for breast cancer: consensus recommendations for The North American Menopause Society and The International Society for the Study of Women's Sexual Health. 2018;25:596-608

26. Gold M, Andriessen A, Bader A, Alinsod R, Freanch ES, Guerette N, et al. Review and clinical experience exploring evidence, clinical efficacy, and safety regaring nonsurgical treatment of feminine rejuvenation. J Cosmet Dermatol 2018;17:289-97

27. FDA Warns Against Use of Energy-Based Devices to Perform Vaginal "Rejuvenation" or Vaginal Cosmetic Procedures: FDA Safety Communication. Available at: https://www.fda.gov/ medical-devices/safety-communications/fdawarns-against-use-energy-based-devices-perform-vaginal-rejuvenation-or-vaginal-cosmetic (last accessed on 10.11.2019)

28. Paraiso MFR, Ferrando CA, Sokol ER, Rardin CR, Matthews CA, Karram MM, et al. A randomized clinical trial comparing vaginal laser therapy to vaginal estrogen therapy in women with genitourinary syndrome of menopause: The VeLVET Trial. Menopause 2019; Doi:10.1097/ GME.0000000000001416

29. Fractional $\mathrm{CO}_{2}$ laser versus promestriene and lubricant in genitourinary syndrome of menopause: a randomized controlled trial. Politano CA, Costa-Paiva L, Aguiar LB, Machado HC, Baccaro LF. Menopause 2019;26:883-40

30. Athanasiou S, Pitsouni E, Grigoriadis T, Zacharakis D, Falagas ME, Salvatore S, et al. Microabaltive fractional $\mathrm{CO}_{2}$ laser for the genitourinary syndrome of menopause: up to 12-month results. Menopause 2019;26:248-55

31. Mothes AR, Runnebaum M, Runnebaum IB. Ablative dual-phase Erbium:YAG laser treatment of atrophy-related vaginal symptoms in postmenopausal breast cancer survivors omitting hormonal treatment. J Cnacer Res Clin Oncol 2018;144:955-60

32. Gambacianni M, Levancini M, Russo E, Vacca L, Simoncini T, Cervigni M. Long-term effects of vaginal erbium laser in the treatment of genitourinary syndrome of menopause. Climacteric 2018;21:148-52

33. Cruz VL, Steiner ML, Pompei LM, Strufaldi R, Fonseca FLA, Santiago LHS, et al. Randomized, double-blind, placebo-controlled clinical trial for evaluating the efficacy of fractional $\mathrm{CO}_{2}$ laser compared with topical estriol in the treatment of vaginal atrophy in postmenopausal women. Menopause 2018;25:21-8

34. Gaspar A, Brandi H, Gomez V, Luque D. Efficacy 
of Erbium:YAG laser treatment compared to topical estriol treatment for symptoms of genitourinary syndrome of menopause. Lasers Surg Med 2017;49:160-8

35. Athanasiou S, Pitsouni E, Falagas ME, Salvatore $S$, Grigoriadis T. $\mathrm{CO}_{2}$-laser for the genitourinary syndrome of menopause. How many laser sessions? Maturitas 2017;104:24-8

36. Salvatore S, Pitsouni E, Del Del F, Parma M, Athanasiou S, Candiani M. Sexual Function in Women Suffering From Genitourinary Syndrome of Menopause Treated With Fractionated $\mathrm{CO}_{2}$-Laser. Sex Med Rev 2017;5:486-94

37. Pitsouni E, Grigoriadis T, Falagas ME, Slavatore S, Athanasiou S. Laser therapy for the genitourinary syndrome of menopause. A systematic review and meta-analysis. Maturitas 2017;103:78-88

38. Pitsouni E, Grigoriadis T, Falagas M, Tsiveleka A, Salvatore S, Athanasiou S. Microablative fractional $\mathrm{CO}_{2}$ laser for the genitourinary syndrome of menopause: power of 30 or 40 W? Pitsouni E, Grigoriadis T, Flagas M, Tsiveleka A, Salvatore S, Athanasiou S. Lasers Med Sci 2017;32:1865-72

39. Behnia-Willison F, Sarraf S, Miller J, Mohamadi B, Care AS, Lam A, et al. Safety and long-term efficacy of fractional $\mathrm{CO}_{2}$ laser treatment in women suffering from genitourinary syndrome of menopause. Eur J Obstet Gynecol Reprod Biol 2017;213:39-44

40. Sokol ER. Karram MM. Use of a novel fractional $\mathrm{CO}_{2}$ laser for the treatment of genitourinary syndrome of menopause:1-year outcomes. Menopause 2017;24:810-4

41. Pieralli A, Bianchi C, Longinotti M, Corioni S, Auzzi $\mathrm{N}$, Becorpi A, et al. Long-term reliability of fractionated $\mathrm{CO}_{2}$ laser as a treatment for vulvovaginal atrophy (VVA) symptoms. Arch Gynecol Obstet 2017;296:973-8

42. Pitsouni E, Grigoriadis T, Tsiveleka A, Zacharakis D, Salvatore S, Athanasiou S. Microablative fractional $\mathrm{CO}_{2}$-laser therapy and the genitourinary syndrome of menopause: An observational study. Maturitas 2016;94:131-6

43. Sokol ER, Karram MM. An assessment of the safety and efficacy of fractional $\mathrm{CO}_{2}$ laser system for the treatment of vulvovaginal atrophy. Menopause 2016;23:1102-7

44. Gambacianni M, Levancini M, Cervigni M. Vaginal erbium laser: the second-generation thermotherapy for the genitourinary syndrome of menopause. Climacteric 2015;18:757-63

45. Salvatore S, Athanasiou S, Candiani M. The use of pulsed $\mathrm{CO}_{2}$ lasers for the treatment of vulvovaginal atrophy. Curr Opin Obstet Gynecol 2015;27:504-8

46. Salvatore S, Nappi RE, Parma M, Chionna R, Lagona $\mathrm{F}$, Zerbinati N, et al. Sexual function after fractional microablative $\mathrm{CO}_{2}$ laser in women with vulvovaginal atrophy. Climacteric 2015;18:219-25

47. Salvatore S, Nappi RE, Zerbinati N, Calligaro A, Ferrero S, Origoni M, et al. A 12-week treatment with fractional $\mathrm{CO}_{2}$ laser for vulvovaginal atrophy: a pilot study. Climacteric 2014;17:363-9

48. Athanasiou S, Pitsouni E, Antonopoulou S, Zacharakis D, Salvatore $S$, Falagas ME, et al. The effect of microablative fractional $\mathrm{CO}_{2}$ laser on vaginal flora of postmenopausal women. Climacteric 2016;19:512-8

49. Salvatore S, Franca K, Lotti T, Parma M, Palmieri $S$, Candiani M, et al. Early Regenerative Modifications of Human Postmenopausal Atrophic Vaginal Mucosa Following Fractional $\mathrm{CO}_{2}$ Laser Treatment. Open Access Maced J Med Sci 2018;19:6-14

50. Zerbinati N, Serati M, Origoni M, Candiani M, Iannitti T, Salvatore $S$, et al. Microscopic and ultrastructural modifications of postmenopausal atrophic vaginal mucosa after fractional carbon dioxide laser treatment. Lasers Med Sci 2015;30:429-36

51. Lapii GA, Yakovlena AY, Neimark AI. Structural Reorganization of the Vaginal Mucosa in Stress Uri- 
nary Incontinence under Conditions of Er:YAG Laser Treatment. Bull Exp Biol Med 2017;162:510-4

52. Vanaman Wilson MJ, Bolton J, Jones IT, Wu DC, Calamae A, Goldman MP. Histologic and clinical changes in vulvovaginal tissue after treatment with a transcutaneous temperature-controlled radiofrequency device. Dermatol Surg 2018;44:705-13

53. Kamilos MF, Borrelli CL. New therapeutic option in genitourinary syndrome of menopause: pilot study using microablative fractional radiofrequency. Einstein (Sao Paulo) 2017;15:445-51

54. Leibaschoff G, Gonzales Izasa P, Cardona JL, Miklos JR, Moore RD. Transcutaneous temperaturecontrolled radiofrequency for the treatment of menopausal vaginal/genitourinary symptoms. Surg Technol Int. 2016;26:29:149-159.

55. Alinsod RM. Temperature controlled radiofrequency for vulvovaginal laxity. Prime. 2015;3:16-21.
56. Krychman M, Rowan CG, Allan BB, DeRogatis L, Durbin S, Yacoubian A, et al. Effect of Single-Treatment, Surface-Cooled Radiofrequency Therapy on Vaginal Laxity and Female Sexual Function: The VIVEVE I Randomizes Controlled Trial. J Sex Med 2017;14:215-225

57. Krychman M, Rowan CG, Allan BB, Durbin S, Yacoubian A, Wilkerson D. Effect of Single-Session, Cryogen-Cooled Monopolar Radiofrequency Therapy on Sexual Function in Women with Vaginal Laxity: The VIVEVE I Trial. J Womens Health (Larchmt) 2018;27:297-304

58. https://hironic.com/p/Ultra\%20Vera (last accessed on 10.11.2019)

Received 28-11-2019

Revised 5-12-2019

Accepted 9-12-2019 